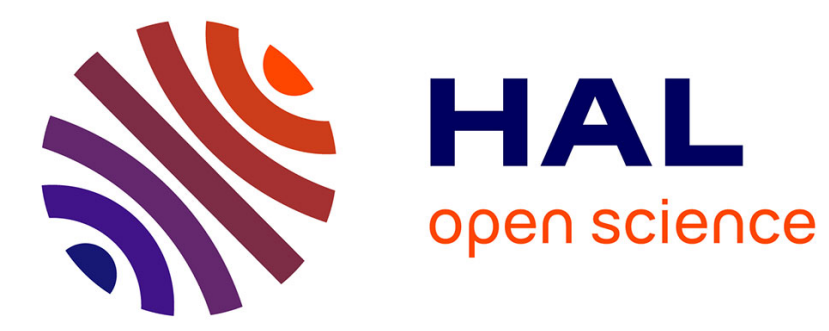

\title{
Four-photon ionization dynamics of xenon
}

\author{
C. Bordas, M. Dyer, T. Fairfield, M. Saeed, H. Helm
}

\section{To cite this version:}

C. Bordas, M. Dyer, T. Fairfield, M. Saeed, H. Helm. Four-photon ionization dynamics of xenon. Journal de Physique IV Proceedings, 1994, 04 (C4), pp.C4-647-C4-650. 10.1051/jp4:19944172 . jpa00252630

\section{HAL Id: jpa-00252630 https://hal.science/jpa-00252630}

Submitted on 1 Jan 1994

HAL is a multi-disciplinary open access archive for the deposit and dissemination of scientific research documents, whether they are published or not. The documents may come from teaching and research institutions in France or abroad, or from public or private research centers.
L'archive ouverte pluridisciplinaire HAL, est destinée au dépôt et à la diffusion de documents scientifiques de niveau recherche, publiés ou non, émanant des établissements d'enseignement et de recherche français ou étrangers, des laboratoires publics ou privés. 


\title{
Four-photon ionization dynamics of xenon
}

\author{
C. BORDAS*, M.J. DYER, T.A. FAIRFIELD, M. SAEED and H. HELM
}

Molecular Physics Laboratory, SRI International, 333 Ravenswood Avenue, Menlo Park, CA 94025, U.S.A.

* Laboratoire de Spectrométrie Ionique et Moléculaire, URA 171 du CNRS, Université Lyon I, Bât. 205, 43 Bd du 11 Novembre 1918, 69622 Villeurbanne cedex, France

\begin{abstract}
Three-photon resonance enhanced four-photon ionization of xenon at wavelengths between 313 and $371 \mathrm{~nm}$ has been investigated by means of a novel photoelectron imaging spectrometer [1]. Photoelectron images provide a direct view of the squared angular wave functions of the free electrons as well as their energy distribution. Preferential formation of $\mathrm{Xe}^{+}\left({ }^{2} \mathrm{P}_{1 / 2}\right)$ is observed when intermediate $\mathrm{ns}[3 / 2](\mathrm{J}=1)$ states belonging to the ${ }^{2} \mathrm{P}_{3 / 2}$ core series are excited in the three-photon step. The opposite behaviour is observed for the 7s' [1/2] $(\mathrm{J}=1)$ state. For the nd states the abundance of photoions in each fine siructure state closely mirrors the core character of the three-photon intermediate.
\end{abstract}

\section{I - Introduction.}

There has been recently considerable interest in the study of multiphoton ionization of heavy rare gases in the moderate field regime [2,3] as well as in the strong field regime $[1,4]$. Rare gases atoms are ideal systems in which to study multiphoton ionization processes, owing to the relative simplicity of their electronic structure. However, the detailed interpretation of the observed effects, especially when strong laser fields are used, is often precluded by the complexity of the elementary processes involved. This point has motivated the present study, concerned with the final state distribution and the respective angular distributions of photoelectrons, of twenty six three-photon resonant intermediates that participate in four-photon ionization of xenon. This study has been conducted under moderate laser field in order to avoid high intensities effects such as ac Stark shifts or above threshold ionization. From resonance enhanced four-photon ionization, we expect that detailed information about every intermediate state participating in the multiphoton ionization process can be extracted in order to understand experiments conducted under high laser intensity. The main result of the present work is the following: for nd Rydberg states, the branching between the two ionization channels $\left(\mathrm{Xe}^{+}\left({ }^{2} \mathrm{P}_{3 / 2}\right)\right.$ and $\left.\mathrm{Xe}^{+}\left({ }^{2} \mathrm{P}_{1 / 2}\right)\right)$ mirrors the mixing of core states in the three-photon intermediate, while a major deviation from this rule appears for the ns [3/2] $(\mathrm{J}=1)$ states.

\section{II - Experimental and Results.}

The experiments are carried out at near-threshold intensities $\left(1-5 \mathrm{GW} / \mathrm{cm}^{2}\right)$ using a nanosecond dye laser, linearly polarized, tuned to selectively excite the $\mathrm{J}=1$ and $\mathrm{J}=3$ resonances of xenon lying below the first ionization limit. A photoelectron imaging spectrometer [1] was used to measure the relative ionization yield as well as the angular distribution of photoelectrons in the ionization channels $\left(\mathrm{Xe}^{+}\left({ }^{2} \mathrm{P}_{3 / 2}\right)+\mathrm{e}^{-}\right)$and $\left(\mathrm{Xe}^{+}\left({ }^{2} \mathrm{P}_{1 / 2}\right)+\mathrm{e}^{-}\right)$. The principle of the imaging spectrometer is the following (see figure 1): when photoelectrons are formed in a field-free region with a given energy $W$, at a point source at $t=0$, they are later found on the surface of a sphere that expands proportionally to $\left(\mathrm{W}^{1 / 2} \mathrm{t}\right)$. If we apply a constant electric field across the ionization region, the electrons are projected onto a two-dimensional position sensitive detector (tandem micro-channel plates). This results in a circular image of electron impacts with a diameter proportional to the square root of the initial kinetic energy, and a filling pattern corresponding to the spatial distribution of the photoelectrons immediately after ionization. The 
Figure 1: Schematic of the photoelectron imaging spectrometer.

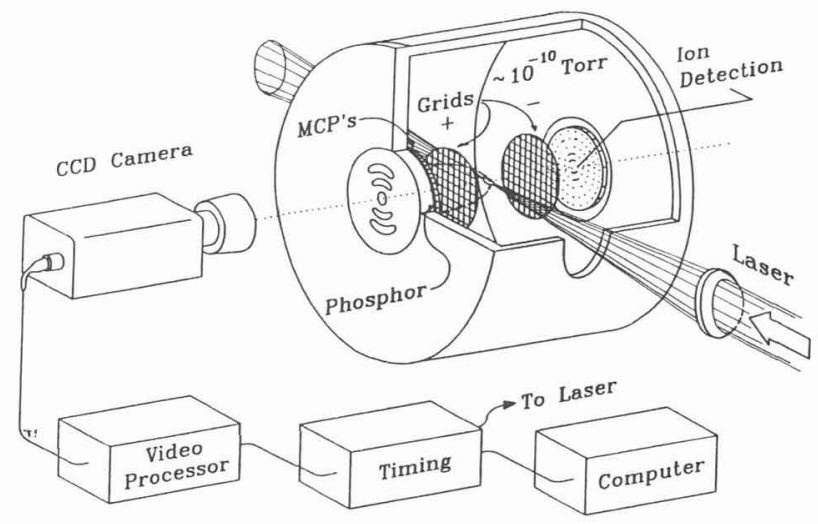

electrons exiting the detector are then accelerated to about $7 \mathrm{keV}$ to impact on a phosphor screen. The screen phosphorescence is subsequently recorded via a CCD camera and digitized in a micro-computer. Many electrons have to be projected in order to generate a statistically meaningful image. This is accomplished by digital summing of electron impact positions from several thousand laser shots, each shot capturing typically 10 to 100 electrons.The electron emission pattern is rotationally symmetric around the laser polarization axis. Therefore, the planar image recorded on a detector parallel to the laser polarization and propagation, can be deconvoluted by an Abel inversion giving directly the initial velocity and angular distribution of the photoelectrons.
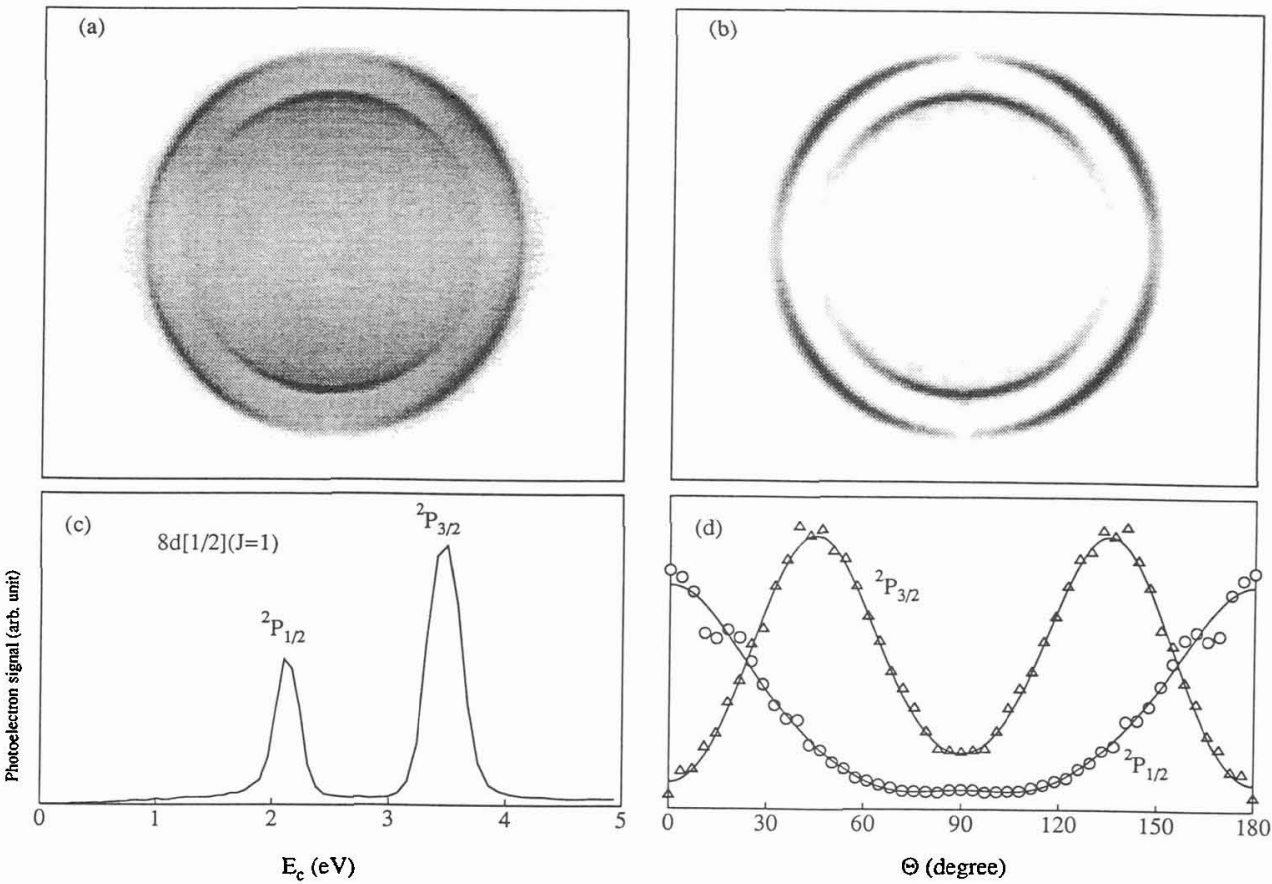

Figure 2: Typical experimental results obtained with the $8 d[1 / 2](J=1)$ intermediate. (a) raw image, (b) Abel inverted image, (c) photoelectron energy spectrum, (d) angular distributions. 
Electrons ejected in the ground state ionization channel $\left.\left(\mathrm{Xe}^{+}\left({ }^{2} \mathrm{P}_{3 / 2}\right)+\mathrm{e}^{-}\right)\right)$appear with an energy higher by $1.31 \mathrm{eV}$ (fine structure) than electrons ejected in the first excited ionization channel $\left.\left(\mathrm{Xe}^{+}\left({ }^{2} \mathrm{P}_{1 / 2}\right)+\mathrm{e}^{-}\right)\right)$. Therefore, two concentric circular images appear on the screen. An example of a raw experimental data recorded with the $8 \mathrm{~d}[1 / 2](J=1)$ intermediate $(\lambda=318.375 \mathrm{~nm})$ is shown in figure 2-a. The corresponding Abel-inverted image is presented in figure 2-b. The analysis of this image provides the photoelectron energy spectrum (2-c), and the angular distribution in both ionization channels (2-d). Note that the detection efficiency of our spectrometer is independent of the initial kinetic energy. This allows us to determine precisely the branching ratios between both ionization channels. In figure 2, we note substantially different angular emission patterns in the final channels. Electrons populating the ${ }^{2} \mathrm{P}_{1 / 2}$ channel appear preferentially along the polarization axis, while those populating the ${ }^{2} \mathrm{P}_{3 / 2}$ channel are emitted preferentially at an angle of 45 degrees with respect to the polarization axis. This drastic difference in angular distribution is also observed for the $7 \mathrm{~s}[3 / 2](\mathrm{J}=1), 8 \mathrm{~s}[3 / 2](\mathrm{J}=1)$, and $7 \mathrm{~s}^{\prime}[1 / 2](\mathrm{J}=1)$ states, and to a lesser extent for the $9 \mathrm{~s}[3 / 2](\mathrm{J}=1)$ state. Figure 3 summarizes typical results concerning branching ratios and angular distributions.

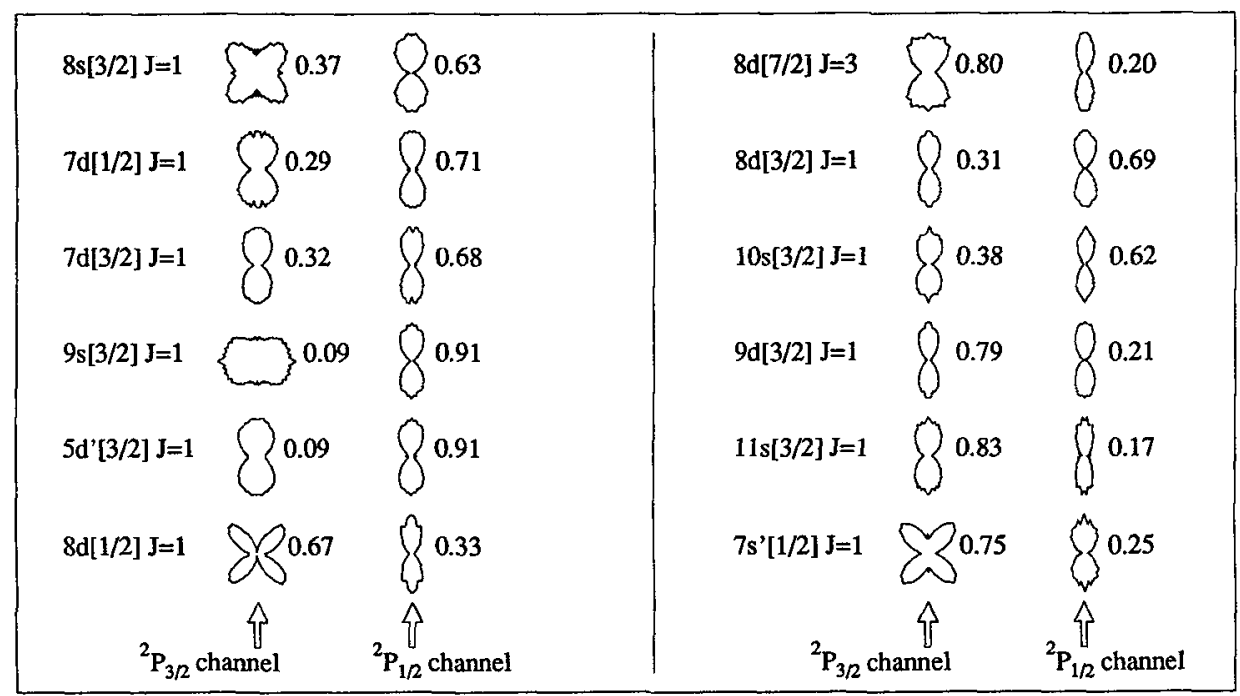

Figure 3: Angular distributions and branching ratios in both ionization channels for 12 selected intermediate states. The laser is polarized along the vertical axis.

\section{III - Discussion.}

The analysis of the angular distributions of photoelectrons $[5,6]$ is beyond the scope of this paper and will be treated elsewhere. Let us focus on the branching ratios among the two fine structure channels. At a first level of approximation, one may assume that the branching into both continua should mimic the configuration mixing in the Rydberg state from which ionization takes place. This configuration mixing can be evaluated in the framework of the Multichannel Quantum Defect Theory (MQDT) [7] using the semi-empirical parameters determined previously [6,8]. The explicit form of the transition moment relevant to the four-photon process involves summation over an infinite number of virtual intermediate states but is dominated by the resonant term. The resonant term itself includes angular terms and matrix elements of the dipolar operator between all channels present in the intermediate state, and all possible channels in the continuum ( $\mathrm{J}=0$ and 2 continua for $\mathrm{J}^{\prime}=1$ Rydberg state, 
$\mathrm{J}=2$ and 4 for $\mathrm{J}^{\prime}=3$ ). Consequently, the configuration mixing in the Rydberg state from which ionization takes place is only at a first approximation proportional to the branching in the respective ${ }^{2} \mathrm{P}_{1 / 2}$ or ${ }^{2} \mathrm{P}_{3 / 2}$ continua. In figure 4, we compare the fraction of electrons ejected in the ${ }^{2} \mathrm{P}_{1 / 2}$ ionization channel to the fraction of ${ }^{2} \mathrm{P}_{1 / 2}$ core in the Rydberg state according to the MQDT calculation. We see that the branching ratios for the nd and nd' intermediates are closely related to the intermediate state core-character predicted by the MQDT treatment. On the other hand, there is a large discrepancy between experimental and theoretical results for the ns and ns' series. The strong deviation in branching from what is expected on the basis of the intermediate-state mixing must lie with the one-photon ionization process that projects the intermediate state into the continuum. A likely origin for the observed deviation is the appearance of a Cooper-type minimum in the cross section of the pure s-type contribution of the intermediate-state wavefunction. Detailed calculations of the ionization process should be able to explain these observations. Such calculations are currently under way $[9,10]$.
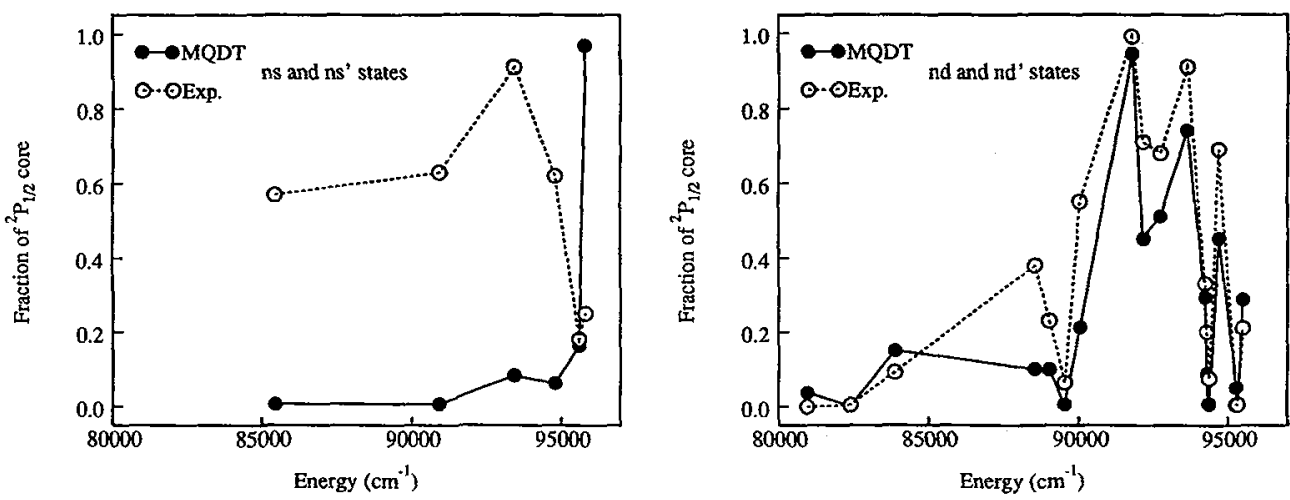

Figure 4: Comparison between experimental branching in the ${ }^{2} P_{1 / 2}$ ionization channel, and ${ }^{2} p_{1 / 2}$ core character of the intermediate for ns and ns' states (left), and nd and nd' states (right).

This research was funded by the National Science Foundation under grant PHY-9249199. T. A. F. acknowledges support through the NSF REU site grant at SRI (NSF - PHY - 9300247).

\section{References:}

[1] H. Helm, N. Bjerre, M.J. Dyer, D.L. Huestis, and M. Saeed, Phys. Rev. Lett. 70,3221 (1993).

[2] S. T. Pratt, P.M. Dehmer, and J.L. Dehmer, Phys. Rev. A 35, 3793 (1987).

[3] K. Sato, Y. Achiba, and K. Kimura, J. Chem. Phys. 8057 (1984).

[4] M.D. Perry, and O.L. Landen, Phys. Rev. A 38,2815 (1988).

[5] P. Gangopadhyay, X. Tang, P. Lambropoulos, and R. Shakeshaft, Phys. Rev. A 34, 2998 (1986).

[6] A. L'Huillier, X. Tang, and P. Lambropoulos, Phys. Rev. A 39,1112 (1989).

[7] K.T. Lu, Phys. Rev. A 4, 579 (1971).

[8] J. Geiger, Z. Physik A 282, 129 (1977).

[9] T. Nakajima, and P. Lambropoulos (private communication).

[10]K. Kulander (private communication). 